\title{
A 500 pc filamentary gas wisp in the disk of the Milky Way
}

\author{
Guang-Xing Li, Friedrich Wyrowski, Karl Menten, and Arnaud Belloche
}

\author{
Max-Planck Institut für Radioastronomie, Auf dem Hügel, 69, 53121 Bonn, Germany \\ e-mail: gxli@mpifr-bonn.mpg.de
}

Received 31 July 2013 / Accepted 27 September 2013

\begin{abstract}
Star formation occurs in molecular gas. In previous studies, the structure of the molecular gas has been studied in terms of molecular clouds, but has been overlooked beyond the cloud scale. We present an observational study of the molecular gas at $49.5^{\circ}<l<52.5^{\circ}$ and $-5.0 \mathrm{~km} \mathrm{~s}^{-1}<v_{\mathrm{lsr}}<17.4 \mathrm{~km} \mathrm{~s}^{-1}$. The molecular gas is found in the form of a huge $(\gtrsim 500 \mathrm{pc})$ filamentary gas wisp. This has a large physical extent and a velocity dispersion of $\sim 5 \mathrm{~km} \mathrm{~s}^{-1}$. The eastern part of the filamentary gas wisp is located $\sim 130 \mathrm{pc}$ above the Galactic disk (which corresponds to $1.5-4$ e-folding scale-heights), and the total mass of the gas wisp is $\gtrsim 1 \times 10^{5} M_{\odot}$. It is composed of two molecular clouds and an expanding bubble. The velocity structure of the gas wisp can be explained as a smooth quiescent component disturbed by the expansion of a bubble. That the length of the gas wisp exceeds by much the thickness of the molecular disk of the Milky Way is consistent with the cloud-formation scenario in which the gas is cold prior to the formation of molecular clouds. Star formation in the filamentary gas wisp occurs at the edge of a bubble (G52L nebula), which is consistent with some models of triggered star formation.
\end{abstract}

Key words. ISM: clouds - ISM: bubbles - ISM: kinematics and dynamics - stars: formation - turbulence

\section{Introduction}

Molecular clouds belong to the densest and coldest parts of the Milky Way interstellar medium (Field et al. 1969; McKee \& Ostriker 1977). Shielded from interstellar radiation fields, they provide conditions necessary for star formation to take place. Observationally, molecular clouds exhibit a complicated, irregular, and filamentary morphology (Bally et al. 1987; Schneider \& Elmegreen 1979; Williams et al. 2000; Goldsmith et al. 2008; Men'shchikov et al. 2010), and (sub)millimeter-line observations of molecular clouds suggest that the gas in the clouds is moving supersonically. Consensus has not been reached concerning the origin and nature of molecular clouds.

It must be recognized that molecular gas is just one of the phases of the Milky Way interstellar medium, and its evolution is determined by many processes that occur in the disk. To understand it, we must also look into the large-scale structure of the multi-phased interstellar medium, and understand the cloud evolution within this context.

Both observational and theoretical approaches have been taken in this direction. Observationally, the distribution of molecular gas in nearby galaxies can be accessed through millimeter line mapping (e.g. Schinnerer et al. 2013), from which structures such as spiral arms, filaments, and spurs can be identified. Theoretically, the structure of the multi-phased interstellar medium in a galactic disk has been studied through simulating the whole disk with different approaches (Kim \& Ostriker 2002; Shetty \& Ostriker 2006; Tasker 2011; Dobbs et al. 2011; Van Loo et al. 2013), complemented by analytical calculations (Lee \& Shu 2012). It was found that filaments or spurs can be created through the combination of gravitational instability of a galactic disk, galactic shear, and frequent

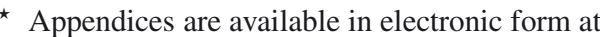
http://www . aanda.org
}

encounters/agglomeration between molecular clouds (Pringle et al. 2001; Dobbs \& Pringle 2013).

In the Milky Way, studies of the structure of molecular gas have been confined spatially to the molecular cloud scale or have been limited to the structure of the spiral arms (Larson 1981; Solomon et al. 1987; Rathborne et al. 2009; Roman-Duval et al. 2009, 2010). This is partly due to the complicated morphology of molecular gas and partly due to the superposition of the emission of molecular gas from different structures along the line of sight. In spite of these difficulties, it is of both observational and theoretical interest to identify large, coherent molecular structures in the Milky Way apart from the spiral arms, since these structures are natural tracers of the large-scale gas circulation in the Milky Way disk. In contrast to the extragalactic case, where we are limited by the resolution and sensitivity of the telescopes (and the filtering of interferometers), for our Milky Way it is possible to study the kinematics of the molecular gas and the associated star formation with in more detail.

The Milky Way interstellar medium has long been thought to be dynamic. Shells and rims are generally found in the disks of the Milky Way and other galaxies (Churchwell et al. 2006). It has been proposed that the expansion of $\mathrm{H}$ II regions, which creates shells and rims, can collect the interstellar medium into a gravitationally unstable state (Elmegreen \& Lada 1977; Whitworth et al. 1994; Whitworth \& Francis 2002), and trigger star formation. The expansion of the bubbles can also energize the interstellar medium of the Milky Way efficiently (Norman \& Ferrara 1996; Mac Low \& Klessen 2004).

In this work, we present an observational study of the region at $49.5^{\circ}<l<52.5^{\circ}$ in the Milky Way. The molecular gas in the region exhibits a high degree of coherence, and forms a filamentary gas wisp (gas filament) with a length of $\sim 3^{\circ}$. The eastern part of the filamentary gas wisp sits at the edge of a bubble and is located at $\sim 0.75^{\circ}$ above the Galactic plane. This eastern part is listed in the context of infrared bubbles as one of the "favorites 
of the Milky-Way-Project volunteers" (Simpson et al. 2012), and it was studied in terms of the G52L nebula by Bania et al. (2012), who claimed that it may be the largest single $\mathrm{H}$ II region in the Milky Way. Based on several estimations (Watson et al. 2003; Anderson \& Bania 2009; Roman-Duval et al. 2010; Bania et al. 2012), the filamentary gas wisp has a distance of $9.77 \mathrm{kpc}$, which implies a physical length of $\gtrsim 500 \mathrm{pc}$. This is $\sim 5$ times longer than the Nessie Nebula reported by Jackson et al. (2010) ${ }^{1}$. The physical length of the filamentary gas wisp exceeds by much the size of a molecular cloud, and this filamentary gas wisp is by far the largest coherent molecular structure identified in the Milky Way. It exhibits a coherent velocity structure, and is composed of several molecular structures, including two molecular clouds and one expanding bubble structure. We present observations and an analysis of the region (Sects. 2, 3), followed by a detailed discussion focusing on the implications on the dynamics of the Milky Way interstellar medium and the life cycle of molecular gas (Sect. 4). In Sect. 5 we conclude.

\section{Archival data}

We obtained $3.6 \mu \mathrm{m}$ and $8 \mu \mathrm{m}$ data from the GLIMPSE project (Benjamin et al. 2003), which is a fully sampled, confusionlimited, four-band near-to-mid infrared survey of the inner Galactic disk. We obtained $24 \mu \mathrm{m}$ data from the MIPSGAL project (Carey et al. 2009), which is a survey of the Galactic disk with the MIPS instrument on Spitzer at $24 \mu \mathrm{m}$ and $70 \mu \mathrm{m}$.

We obtained ${ }^{13} \mathrm{CO}(1-0)$ molecular line data $\left(v_{0}=110.2 \mathrm{GHz}\right)$ from the Galactic Ring Survey (Jackson et al. 2006), which is a survey of the Milky Way disk with the SEQUOIA multipixel array on the Five College Radio Astronomy Observatory $14 \mathrm{~m}$ telescope, and covers a longitude range of $18^{\circ}<l<55.7^{\circ}$ and a latitude range of $|b|<1^{\circ}$ with a spatial resolution of $46^{\prime \prime}$.

\section{Results}

\subsection{Region}

Figure 1 shows the Spitzer three-color image of the region from $49.5^{\circ}<l<52^{\circ}$. The overlaid contours show the molecular gas traced by ${ }^{13} \mathrm{CO}(1-0)$. The $\mathrm{CO}$ emission in all the panels is integrated within $-5.0 \mathrm{~km} \mathrm{~s}^{-1}<v_{\mathrm{lsr}}<17.4 \mathrm{~km} \mathrm{~s}^{-1}$. Several features can be identified. At $51.5^{\circ}<l<52.5^{\circ}$, there is a bubble with a radius of $\sim 1^{\circ}$ (G52L nebula, Bania et al. 2012). The molecular gas is situated to the north of the bubble. At $51^{\circ}<l<52.5^{\circ}$, the molecular gas is organized in the form of two molecular clouds (G052.24+00.74 and G051.69+00.74). The cloud G052.24+00.74 has a roundish shape. This cloud is connected with another molecular cloud, G051.69+00.74. This cloud has a more elongated geometry, and star formation occurs only at its eastern part.

At $49.5^{\circ}<l<50^{\circ}$, there is noticeable contamination from gas with $5.2 \mathrm{~km} \mathrm{~s}^{-1}<v_{\text {lsr }}<7.2 \mathrm{~km} \mathrm{~s}^{-1}$ (see the red arrows in Fig. A.1). The contaminating gas has an extremely narrow line width $\left(\lesssim 0.5 \mathrm{~km} \mathrm{~s}^{-1}\right)$ and tends to spread along the spatial direction. This makes it easily distinguishable from the emission from the gas filament. This narrow line width implies that the emission comes from a close-by cloud. This is supported by the fact that the contaminating gas has a more diffuse morphology (see Appendix A for ${ }^{13} \mathrm{CO}(1-0)$ channel maps of the region).

\footnotetext{
1 Note that Goodman et al. (2013) report a much larger length of "many hundreds of pc" for the Nessie nebula. See also http:// milkywaybones.org/ for more details.
}

This distinction is similar to the supernova remnant G016.050.57 studied in Beaumont et al. (2011).

The two molecular clouds (G052.24+00.74 and G051.69+00.74) have a similar velocity and velocity dispersion: the cloud G052.24+00.74 has $v_{\mathrm{lsr}} \sim 4.6 \mathrm{~km} \mathrm{~s}^{-1}$ and $\delta v \sim$ $2.6 \mathrm{~km} \mathrm{~s}^{-1}$ and the cloud G051.69+00.74 has $v_{\mathrm{lsr}} \sim 3.6 \mathrm{~km} \mathrm{~s}^{-1}$ and $\delta v \sim 3.6 \mathrm{~km} \mathrm{~s}^{-1}$ (Roman-Duval et al. 2010). In the positionposition and position-velocity space, they are connected with some wispy gas filaments (at $l \sim 52^{\circ}$ and $b \sim 0.8^{\circ}$ of the top and middle panels of Fig. 1). The similarity of the velocity dispersions and the proximity of the clouds in position-velocity space imply that the two clouds are physically connected.

It can be readily seen from the ${ }^{13} \mathrm{CO}(1-0)$ emission that this double-cloud system belongs to a large filament (Fig. 1 and Appendix A). The filamentary gas wisp is coherent in both the spatial and the velocity direction, which makes it distinguishable from other molecular structures, for instance the $\sim 50 \mathrm{~km} \mathrm{~s}^{-1}$ clouds (Appendix B). Seen from the middle panel of Fig. 1, the filamentary gas wisp extends from $l=49.5^{\circ}$ to $l=52.5^{\circ}$, which implies an angular extent of $\gtrsim 3^{\circ}$. Seen from the bottom panel of Fig. 1, the filamentary gas wisp has a limited velocity range of $\sim 22 \mathrm{~km} \mathrm{~s}^{-1}\left(-5.0 \mathrm{~km} \mathrm{~s}^{-1}<v_{\mathrm{lsr}}<17.4 \mathrm{~km} \mathrm{~s}^{-1}\right)$. Similar to the double-cloud system, all the molecular gas in the filamentary gas wisp has a similar velocity dispersion. At $49.5^{\circ}<l<50.5^{\circ}$, the filamentary gas wisp seems to be split in both the positionposition and position-velocity maps (middle and bottom panels of Fig. 2). This coincides with the presence of a bubble in the infrared band. To summarize, the filamentary gas wisp is composed of two molecular clouds and one bubble.

Star formation occurs in different parts of the filamentary gas wisp. Star formation in molecular clouds can be conveniently traced by $24 \mu \mathrm{m}$ emission, which originates from the dust heated by newly-born stars. In the Spitzer image, this appears as red regions (Fig. 1 top and Fig. 3). In the cloud pair G052.24+00.74 and G051.69+00.74, several star-forming sites can be identified (Fig. 3) based on the Spitzer $24 \mu \mathrm{m}$ emission, three of which are currently hosting compact $\mathrm{H}$ II regions (Lockman 1989; Urquhart et al. 2009). At 51.5 $<l<52.5^{\circ}$, all the star-forming sites are located at the edge of the G52L bubble.

\subsection{Distance and size of the filament}

The distance to the region has been estimated by several authors. Without trigonometric parallaxes, the distance to the region can be determined with the kinematic method. One key step in determining the kinematic distance is to resolve the kinematic distance ambiguity.

There are different ways to resolve the ambiguity. Distance of the filamentary gas wisp can be determined by studying the distance to molecular clouds and $\mathrm{H}$ II regions that belong to the filament. Using HI self-absorption, Roman-Duval et al. (2010) found that the molecular clouds G052.24+00.74 and $\mathrm{G} 051.69+00.74$ are located at the far distance. Using the $\mathrm{H}_{2} \mathrm{CO}$ absorption line, Watson et al. (2003) found that the $\mathrm{H}$ II region G52.23+0.74 is located at the far distance. Recently, Anderson \& Bania (2009) and Bania et al. (2012) studied the distance to the H II regions G052.201+0.752 and G052.259+0.700 with HI emission/absorption method, and again found that they are at the far distance. Therefore we conclude that the filamentary gas wisp is located at the far distance, which is approximately $9.8 \mathrm{kpc}$. This suggests a galactocentric distance of $8.2 \mathrm{kpc}$, and the filamentary gas wisp probably resides in or around the Perseus arm. 

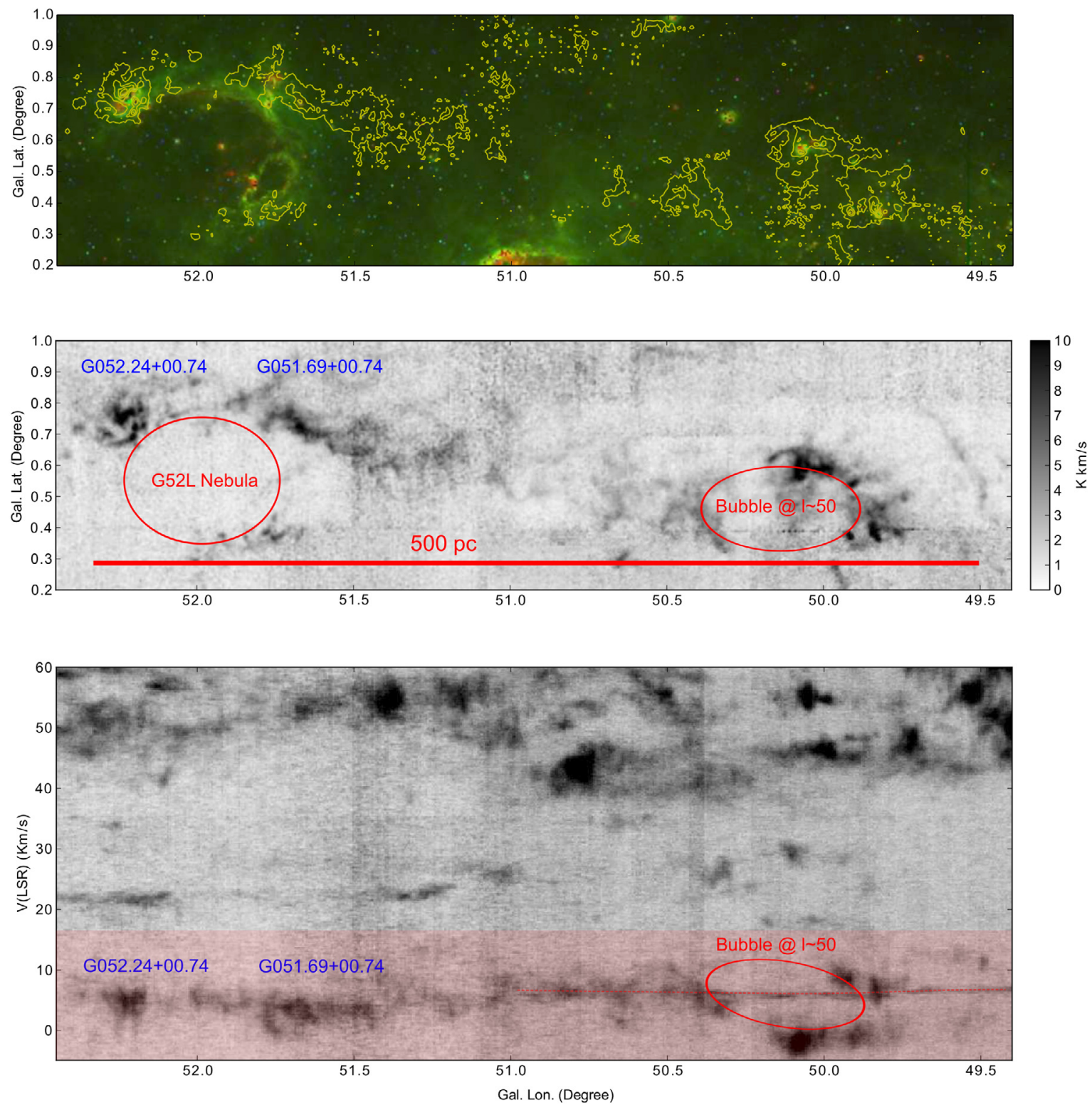

Fig. 1. Top panel: Spitzer GLIMPSE (Benjamin et al. 2003) and MIPSGAL (Carey et al. 2009) three-color image of the region. Red: $24 \mu \mathrm{m}$, green: $8 \mu \mathrm{m}$, blue: $3.6 \mu \mathrm{m}$. Overlaid contours are the velocity-integrated ${ }^{13} \mathrm{CO}(1-0)$ emission $\left(-5.0 \mathrm{~km} \mathrm{~s}^{-1}<v_{\mathrm{lsr}}<17.4 \mathrm{~km} \mathrm{~s}{ }^{-1}\right)$ from the Galactic Ring Survey (Jackson et al. 2006). Contours correspond to 3.5, 7.3, 11.2, $15 \mathrm{~K} \mathrm{~km} \mathrm{~s}^{-1}$. Middle panel: velocity-integrated ${ }^{13} \mathrm{CO}(1-0)$ map of the region integrated within $-4.95 \mathrm{~km} \mathrm{~s}^{-1}<v_{\mathrm{lsr}}<17.36 \mathrm{~km} \mathrm{~s}^{-1}$. A scale bar of $500 \mathrm{pc}$ is added assuming a kinematic distance of $9.8 \mathrm{kpc}$. Bottom panel: Galactic-latitude-integrated ${ }^{13} \mathrm{CO}(1-0)$ position-velocity map of the region (integrated from $-0.2^{\circ}<b<1^{\circ}$ ). The clouds G052.24+00.74, $\mathrm{G} 051.69+00.74$, the bubble at $l \sim 50^{\circ}$, and the G52L nebula (Bania et al. 2012) are indicated in the middle and bottom panels. The velocity range we used to produce the ${ }^{13} \mathrm{CO}(1-0)$ integrated intensity map is indicated in the bottom panel as the red shaded region. The emission at $5.2 \mathrm{~km} \mathrm{~s} \mathrm{~s}^{-1}<$ $v_{\mathrm{lsr}}<7.2 \mathrm{~km} \mathrm{~s}^{-1}$ is due to contamination from a different molecular cloud, and some of the emission lies on the red dashed line. This component has a smaller line width $\lesssim 0.5 \mathrm{~km} \mathrm{~s}^{-1}$, which implies that the contamination comes from a close-by cloud. This is supported by its apparent diffuse morphology. Channel maps of the region are provided in Appendix A.

Accordingly, the filamentary gas wisp we identified has a spatial extent of $\sim 500 \mathrm{pc}$. If the filamentary gas wisp follows the spiral structure, it is probably angled $\sim 45^{\circ}$ to our line of sight, and therefore probably has a deprojected length a factor of $\sqrt{2}$ longer. Therefore we conclude that the filamentary gas wisp has a length of $\gtrsim 500 \mathrm{pc}$. It is one the of the largest coherent molecular structures in the Milky Way apart from the spiral arms and the molecular ring. The total mass of the filamentary gas wisp can be estimated using the ${ }^{13} \mathrm{CO}(1-0)$ emission. To do this, we integrated over the region with the line-ofsight integrated flux $I>3.5 \mathrm{~K} \mathrm{~km} \mathrm{~s}^{-1}$. This corresponds to the first contour in the upper panel of Fig. 1. This mass estimate should be considered as a lower limit since by selecting this threshold we only take the region with a high column density $\left(N_{\mathrm{H}_{2}}>1.75 \times 10^{21} \mathrm{~cm}^{-2}\right)$ into account. Using Eqs. (1)-(3) of Roman-Duval et al. (2010) and assuming an excitation temperature of $10 \mathrm{~K}$, we obtain a total mass of $\sim 1 \times 10^{5} M_{\odot}$ for the whole gas wisp $\left(49.5^{\circ}<l<52.5^{\circ}\right)$. According to Simon et al. (2001), the derived mass is only weakly sensitive to this choice of excitation temperature, and in our case an excitation temperature of $20 \mathrm{~K}$ gives a mass of $\sim 0.6 \times 10^{5} M_{\odot}$.

The two clouds at the eastern part of the filamentary gas wisp have $b \sim 0.74^{\circ}$. Using a kinematic distance of $9.77 \mathrm{kpc}$, the double-cloud system is $\sim 130 \mathrm{pc}$ above the Galactic plane. At 

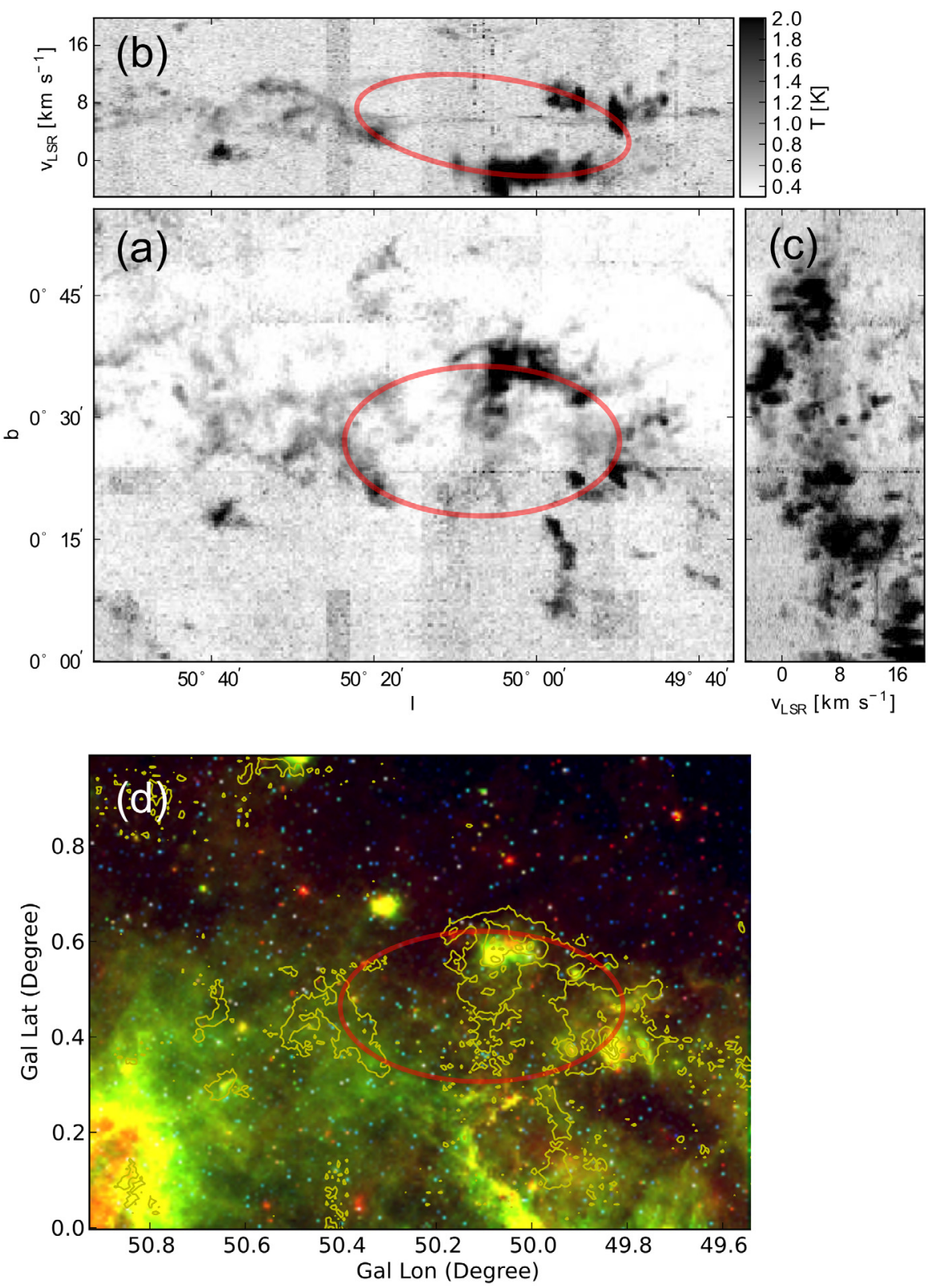

Fig. 2. a) Galactic longitude-latitude map of the peak temperatures of the ${ }^{13} \mathrm{CO}(1-0)$ data cube along the velocity axis. b) Galactic-longitudevelocity map of peak temperatures of ${ }^{13} \mathrm{CO}(1-0)$ along the galactic latitude axis. c) Velocitygalactic-latitude map of peak temperatures of ${ }^{13} \mathrm{CO}(1-0)$ along the galactic longitude axis. d) Spitzer GLIMPSE (Benjamin et al. 2003) and MIPSGAL (Carey et al. 2009) three-color image of the region. Red: $24 \mu \mathrm{m}$, Green: $8 \mu \mathrm{m}$, Blue: $3.6 \mu \mathrm{m}$. Overlaid contours are the velocity-integrated ${ }^{13} \mathrm{CO}(1-0)$ emission from the Galactic Ring Survey (Jackson et al. 2006) $\left(-4.95 \mathrm{~km} \mathrm{~s}^{-1}<v_{\mathrm{lsr}}<17.36 \mathrm{~km} \mathrm{~s}^{-1}\right)$. Contours correspond to $3.5,7.3,11.2,15 \mathrm{~K} \mathrm{~km} \mathrm{~s}^{-1}$. In a) b), and d), the bubble is indicated as a red ellipse. a Galactocentric distance of $\sim 8 \mathrm{kpc}$, the molecular disk of the Milky Way has a FWHM thickness of $90-180$ pc (at 7-8 kpc the FWHM is $\sim 90 \mathrm{pc}$ and at $8-9 \mathrm{kpc}$ the FWHM is $186 \mathrm{pc}$, Nakanishi \& Sofue 2006). This corresponds to an e-folding height of 38-80 pc. Therefore the height of the double-cloud system is about 1.5-4 times the e-folding height of the Galactic disk. The double-cloud system is a unique cloud system that is located far above the Galactic plane. According to Bania et al. (2012), one possible explanation is that the material of the system has been displaced by the expansion of the G52L nebula.

\subsection{The bubble structure at $I \sim 50^{\circ}$}

Figure 2 shows the bubble structure at $l \sim 50^{\circ}$. Its boundary is visible in both the $8 \mu \mathrm{m}$ emission, which traces polycyclic aromatic hydrocarbon (PAHs) and in the ${ }^{13} \mathrm{CO}(1-0)$ emission. The bubble is not easily visible at $24 \mu \mathrm{m}$, which traces hot dust heated by a central star. Because of the apparent absence of the $24 \mu \mathrm{m}$ emission, the bubble structure does not seem to be driven by the expansion of a H II region. This is also supported by the absence of a diffuse H II region in the VGPS (Stil et al. 2006) continuum image.

It is more likely that the bubble structure is driven by the expansion of a supernova. The Spitzer image of the bubble resembles that of several supernova remnants in the Churchwell et al. (2006) catalog. From panel a of Fig. 2, using the kinematic distance of $9.8 \mathrm{kpc}$, we estimate a diameter of $1^{\circ} \sim 180 \mathrm{pc}$, and from panel b of Fig. 2 we estimate a total expansion velocity of $10 \mathrm{~km} \mathrm{~s}^{-1}$. These give an age of $50 \mathrm{Myr}$. The energy of a possible supernova explosion can be estimated through the Sedov-Taylor solution: $E \sim r^{3} v^{2} \rho \sim 0.16 \times 10^{51} \mathrm{erg}$. The energy is consistent with a supernova explosion. Here, a density of $10^{-24} \mathrm{~g} \mathrm{~cm}^{-3}$ is used, which is typical of warm neutral medium (cf. Bocchino et al. 2010).

\section{Discussion}

\subsection{Morphology of the filamentary gas wisp}

This giant molecular structure is among the largest molecular structures studied in the Milky Way ( $2500 \mathrm{pc})$. The physical size 


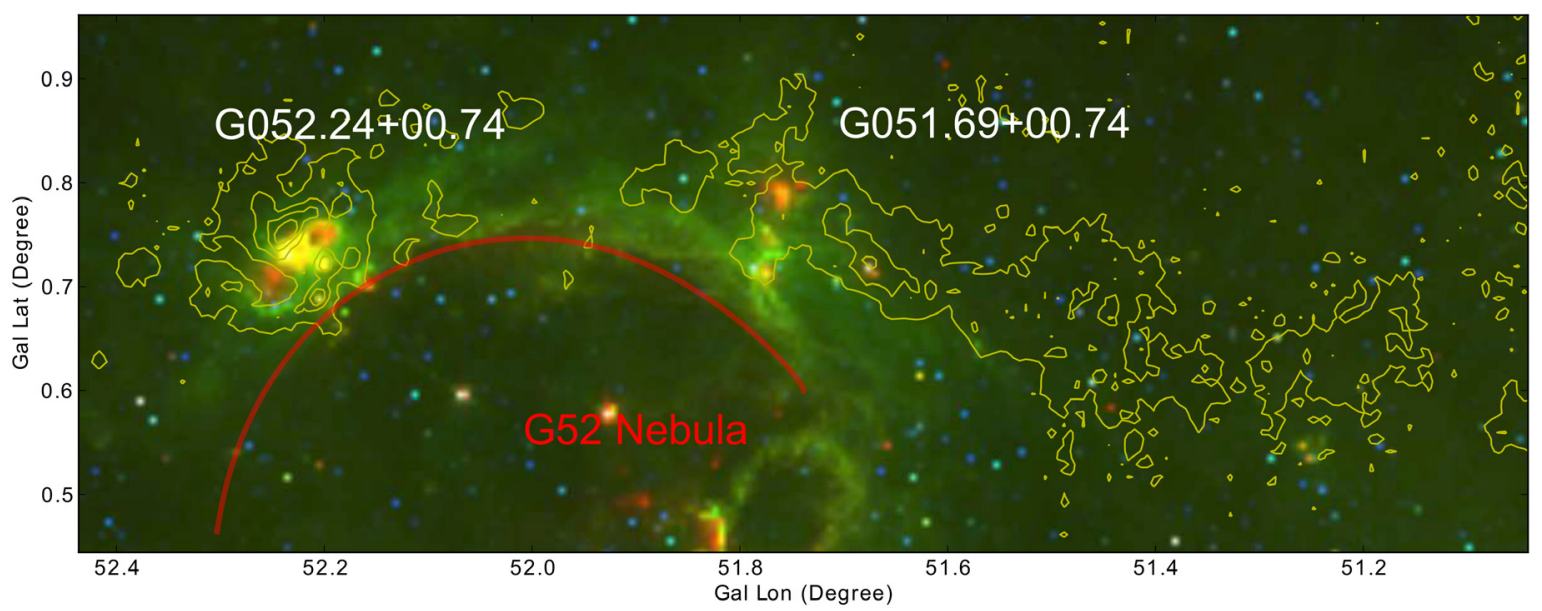

Fig. 3. Spitzer GLIMPSE (Benjamin et al. 2003) and MIPSGAL three-color image of the clouds G052.24+00.74 and G51.69+00.74. Red: $24 \mu \mathrm{m}$, green: $8 \mu \mathrm{m}$, blue: $3.6 \mu \mathrm{m}$. Overlaid contours are the velocity-integrated ${ }^{13} \mathrm{CO}(1-0)$ emission $\left(-4.95 \mathrm{~km} \mathrm{~s}^{-1}<v_{\text {lsr }}<17.36 \mathrm{~km} \mathrm{~s}{ }^{-1}\right)$ from the Galactic Ring Survey (Jackson et al. 2006). Contours correspond to 3.5, 7.3, 11.2, $15 \mathrm{~K} \mathrm{~km} \mathrm{~s}^{-1}$. The cloud G052.24+00.74 and G51.69+00.74 as well as the G52L nebula are indicated.

of the gas filamentary gas wisp is much larger than that of a typical molecular cloud ( 10 pc, Roman-Duval et al. 2010). The velocity dispersion along a single line of sight in the filamentary gas wisp is not significantly different from that of ordinary molecular clouds.

The molecular gas in the filamentary gas wisp is concentrated in the vertical (Galactic-latitude) direction and elongated along the horizontal (Galactic-longitude) direction. At different locations, the filamentary gas wisp exhibits a different width. At $49.5^{\circ}<l<50.5^{\circ}$, the filamentary gas wisp is split in the map, which makes it difficult to define its width. From the map, the cloud G052.24+00.74 appears to be more extended in the vertical direction than the cloud G051.69+00.74. We therefore used its vertical extent as an estimate of the width of the filamentary gas wisp. The vertical extent of the cloud G051.69+00.74 is measured for the region with $I>3.5 \mathrm{~K} \mathrm{~km} \mathrm{~s}^{-1}\left(N_{\mathrm{H}_{2}}>\right.$ $\left.1.75 \times 10^{21} \mathrm{~cm}^{-2}\right)$. This corresponds to the first contour in the upper panel of Fig. 1. We found that the cloud extends from $b \sim 0.65^{\circ}$ to $b \sim 0.82^{\circ}$. From this we estimated a diameter of $30 \mathrm{pc}$, which implies an aspect ratio of $\sim 600 / 30=20$ for the gas wisp. The gas wisp is one of the most elongated molecular structures found in the Milky Way (see also the Nessie nebula, Jackson et al. 2010). The width of the filamentary gas wisp is narrower than the FWHM thickness of the molecular disk of the Milky Way, which is about 90-180 pc (Nakanishi \& Sofue 2006) at a Galactocentric distance of $\sim 8 \mathrm{kpc}$.

Similar large-scale molecular structures have been observed in other galaxies. In spiral galaxies, elongated gas condensations are frequently observed. They can be seen as narrow dark lanes that extend perpendicular to the spiral arms (Lynds 1970; Weaver 1970). The exact definitions of dust lanes or spurs differ in the literature. However, in most cases spurs refer to the objects whose widths are similar to that of spiral arms (Elmegreen 1980). In our case, the filamentary gas wisp should not be termed a spur because its width is narrower than the width of the spiral arms of a typical galaxy, which is 500 pc (Egusa et al. 2011).

In our case, the filamentary gas wisp is about one or two orders of magnitudes longer than ordinary molecular clouds, but is still much narrower than the spurs in galaxies. Therefore we propose that the filamentary gas wisp is a new object that is yet to be classified. Because of this, we termed it a gas wisp in this work to emphasize its elongated morphology.
Even though the thickness of the filamentary gas wisp is similar to the resolution of the PAWS survey (Schinnerer et al. 2013) of M51, filamentary gas wisps of this size would not be detected. This is because the survey is only sensitive to objects with a mass $\gtrsim 1.2 \times 10^{5} M_{\odot}$ and the clouds in the filamentary gas wisp are only $\sim 10^{4} M_{\odot}$. However, similar large-scale gas structures in nearby galaxies are probably suitable targets for ALMA thanks to its improved sensitivity. Nearby face-on galaxies are expected to be excellent sites for studying these gas wisps since lineof-sight confusion can be avoided. A future project at ALMA targeting at the molecular gas in nearby face-on galaxies is expected to resolve similar gas condensations and provide a more complete picture of the structure of molecular gas in galaxies.

\subsection{Implications for the formation of molecular clouds}

The formation and evolution of molecular clouds is one of the fundamental problems in interstellar medium studies. To account for the short formation timescale of molecular clouds, two scenarios have been proposed. The first scenario involves colliding flows. In this scenario, molecular clouds form from diffuse gas (warm neutral medium) collected into a dense phase (cold neutral medium/molecular medium) through colliding flows (Audit \& Hennebelle 2005; Heitsch et al. 2006; Vázquez-Semadeni et al. 2007, 2010; Inoue \& Inutsuka 2012). The molecular gas can form quickly in the converging flows because of dynamically-triggered thermal instability.

The second scenario has been proposed by Pringle et al. (2001) and Dobbs \& Pringle (2013). In this scenario, the gas is already relatively dense and cold prior to becoming a giant molecular cloud. According to Pringle et al. (2001), there is expected to be copious cold gas in the inter-arm regions since the circulation of the molecular gas is a process that occurs at the disk scale. To describe the global circulation of the gas, we divided it into the in-arm phase in which the gas is situated inside the spiral arms, and the inter-arm phase in which the gas is situated in the inter-arm regions. As discussed in Pringle et al. (2001), in the inter-arm phase, the cold gas exists in the form of wisps, and the gas in these wisps will show up as giant molecular clouds during the spiral-arm phase. This has been largely confirmed by the simulations of Dobbs \& Pringle (2013), which track the evolution of single molecular clouds. These authors 
found that molecular clouds begin to disperse as they leave the spiral arm. Due to differential shear, the molecular clouds are transformed into filamentary gas wisps in the inter-arm region. Since the shear occurs at a large scale, we expect to see gas wisps whose physical scale exceeds the thickness of the Milky Way disk. In our case, the physical length of the filamentary gas wisp ( $\gtrsim 500 \mathrm{pc}$ ) is much larger than the scale-height of the Milky Way molecular disk. This is consistent with the cloud-formation scenario by Pringle et al. (2001) and Dobbs \& Pringle (2013). Such large-scale structures are also observed in other numerical simulations of galactic disks (Tan 2000; Kim \& Ostriker 2002; Dobbs \& Bonnell 2006; Dobbs et al. 2006; Shetty \& Ostriker 2006; Tasker \& Tan 2009; Ceverino et al. 2012).

On the other hand, it is difficult to understand the filamentary gas wisp in the converging flow scenario. In this scenario, molecular gas forms from the converging HI gas through the dynamically-triggered thermal instability. As summarized in Dobbs et al. (2012), the sources of the converging flows can be stellar winds or supernovae (Koyama \& Inutsuka 2000; Heitsch \& Hartmann 2008; Ntormousi \& Burkert 2011), turbulence in the interstellar medium (Ballesteros-Paredes et al. 1999), spiral shocks (Leisawitz \& Bash 1982), and gravitational instability. In our case, the filamentary gas wisp cannot be created by converging stellar winds, supernovae, or turbulence in the interstellar medium, since these mechanisms are local and cannot create structures that are larger than the thickness of the Milky Way disk. Spiral shocks and gravitational instability might create conditions favorable for converging flows to occur. However, to access these possibilities we need to simulate converging flows in a galactic context and properly quantify the role of the dynamically-triggered thermal instability in the formation of molecular gas. This task has not been achieved yet. Either the converging flow scenario is unable to explain how filamentary gas wisps form, or our current understanding of converging flows in a galactic context is incomplete.

\subsection{Star formation in the molecular cloud pair G0524.2+00.74 and G051.69+00.74}

It is unclear to what extent molecular clouds are gravitationally bound. Gravity is important at a variety of physical scales during star formation. According to Roman-Duval et al. (2010), the clouds G052.24+00.74 and G051.69+00.74 have virial parameters of 0.29 and 0.33 , respectively. This means that both clouds are gravitationally bound ${ }^{2}$.

Star formation takes place in several sites, which is traced by the $24 \mu \mathrm{m}$ emission in the Spitzer image. As indicated in Fig. 3, all these sites seem to be located at the edge of a bubble (G52L nebula).

The connection between the location of the star-forming sites and the edge of the bubble agrees with the statistical study of Thompson et al. (2012), in which a significant overdensity of young stellar objects toward the edges of the bubbles was found. This is consistent with the collect-and-collapse scenario of triggered star formation (Elmegreen \& Lada 1977; Whitworth et al. 1994).

\section{Conclusions}

We studied a giant coherent molecular structure (a filamentary gas wisp) at $49.5^{\circ}<l<52.5^{\circ}$. The eastern part of the filamentary gas wisp is located $\sim 130$ pc above the Galactic disk

\footnotetext{
2 Here and in Roman-Duval et al. (2010) the virial parameter $\alpha$ of a molecular cloud is the ratio of its virial mass $M_{\text {vir }}$ to its mass.
}

(which corresponds to 1.5-4 e-folding scale-heights), and the total mass of the gas wisp is $\gtrsim 1 \times 10^{5} M_{\odot}$. Apart from the spiral arms and the molecular ring, this is among the largest coherent molecular structures identified in the Milky Way. The velocity structure of the filamentary gas wisp is coherent and smooth at $50.5^{\circ}<l<52.5^{\circ}$, and at $49.5^{\circ}<l<50.5^{\circ}$, the gas wisp is disturbed by a bubble structure. This might be caused by a supernova. The overall velocity structure of the filamentary gas wisp can be understood as a quiescent filamentary gas wisp disturbed by the expansion of a bubble. The eastern part of the filamentary gas wisp is composed of a system of two molecular clouds (G052.24+00.74 and G051.69+00.74) and is located $\sim 130$ pc above the Galactic plane.

Star formation already takes place in several parts of this filamentary gas wisp. In the cloud pair G052.24+00.74 and G051.69+00.74, nearly the entire star formation occurs at the edge of a bubble (G52L nebula, Bania et al. 2012). This is consistent with the collect-and-collapse scenario of triggered star formation (Elmegreen \& Lada 1977; Whitworth et al. 1994), and can be understood in the statistical context of Thompson et al. (2012).

The discovery of this filamentary gas wisp, whose length exceeds the thickness of the molecular disk of the Milky Way, suggests that the formation and evolution of molecular clouds is a phenomenon that occurs at the disk scale. The large physical extent is consistent with the cloud-formation scenario by Pringle et al. (2001) and Dobbs \& Pringle (2013), in which the gas that constitutes the molecular clouds is already relatively cold prior to the cloud formation.

We are currently unable to answer how representative this filamentary gas wisp is in the Milky Way disk. One reason is that we are restricted by the line-of-sight confusion, and the filamentary gas wisp is fragile in nature. In our case, at $49.5^{\circ}<$ $l<50.5^{\circ}$, the filamentary gas wisp is already being destroyed by the expansion of a bubble structure. It is possible that a significant fraction of the gas in the Milky Way exists in this form during at least part of its lifetime. Another difficulty is to properly quantify the coherence of molecular structures beyond the cloud scale. A position-velocity plot of the ${ }^{13} \mathrm{CO}(1-0)$ data from the same region shown in the bottom panel of Fig. 1 reveals filamentary structures at $v_{\mathrm{lsr}} \sim 50 \mathrm{~km} \mathrm{~s}^{-1}$. From a visual inspection we found that these structures are not as coherent as the filamentary gas wisp (see Appendix B for a comparison). In general one cannot yet quantify the coherence of molecular structures in the Milky Way. More studies of the morphology of the molecular gas in both the Milky Way and other galaxies with improved observations and analyses are needed to fully understand the circulation of molecular gas at large scales.

Acknowledgements. Guang-Xing Li is supported for this research through a stipend from the International Max Planck Research School (IMPRS) for Astronomy and Astrophysics at the Universities of Bonn and Cologne. This publication makes use of molecular line data from the Boston UniversityFCRAO Galactic Ring Survey (GRS). The GRS is a joint project of Boston University and Five College Radio Astronomy Observatory, funded by the National Science Foundation under grants AST-9800334, AST-0098562, and AST-0100793. This work is based in part on observations made with the Spitzer Space Telescope, which is operated by the Jet Propulsion Laboratory, California Institute of Technology under a contract with NASA. We thank James Urquhart and Malcolm Walmsley for careful readings of our paper and for many insightful comments. We thank the referee Adam Ginsburg for several thorough and careful reviews of the paper and for his insightful comments.

\section{References}

Anderson, L. D., \& Bania, T. M. 2009, ApJ, 690, 706 Audit, E., \& Hennebelle, P. 2005, A\&A, 433, 1 
Ballesteros-Paredes, J., Hartmann, L., \& Vázquez-Semadeni, E. 1999, ApJ, 527, 285

Bally, J., Lanber, W. D., Stark, A. A., \& Wilson, R. W. 1987, ApJ, 312, L45

Bania, T. M., Anderson, L. D., \& Balser, D. S. 2012, ApJ, 759, 96

Beaumont, C. N., Williams, J. P., \& Goodman, A. A. 2011, ApJ, 741, 14

Benjamin, R. A., Churchwell, E., Babler, B. L., et al. 2003, PASP, 115, 953

Bocchino, F., Bandiera, R., \& Gelfand, J. 2010, A\&A, 520, A71

Carey, S. J., Noriega-Crespo, A., Mizuno, D. R., et al. 2009, PASP, 121, 76

Ceverino, D., Dekel, A., Mandelker, N., et al. 2012, MNRAS, 420, 3490

Churchwell, E., Povich, M. S., Allen, D., et al. 2006, ApJ, 649, 759

Dobbs, C. L., \& Bonnell, I. A. 2006, MNRAS, 367, 873

Dobbs, C. L., \& Pringle, J. E. 2013, MNRAS, 432, 653

Dobbs, C. L., Bonnell, I. A., \& Pringle, J. E. 2006, MNRAS, 371, 1663

Dobbs, C. L., Burkert, A., \& Pringle, J. E. 2011, MNRAS, 413, 2935

Dobbs, C. L., Pringle, J. E., \& Burkert, A. 2012, MNRAS, 425, 2157

Egusa, F., Koda, J., \& Scoville, N. 2011, ApJ, 726, 85

Elmegreen, D. M. 1980, ApJ, 242, 528

Elmegreen, B. G., \& Lada, C. J. 1977, ApJ, 214, 725

Field, G. B., Goldsmith, D. W., \& Habing, H. J. 1969, ApJ, 155, L149

Goldsmith, P. F., Heyer, M., Narayanan, G., et al. 2008, ApJ, 680, 428

Goodman, A. A., Alves, J. F., Beaumont, C., et al. 2013, in Am. Astron. Soc. Meet. Abst., 221, 234.01

Heitsch, F., \& Hartmann, L. 2008, ApJ, 689, 290

Heitsch, F., Slyz, A. D., Devriendt, J. E. G., Hartmann, L. W., \& Burkert, A. 2006, ApJ, 648, 1052

Inoue, T., \& Inutsuka, S.-I. 2012, ApJ, 759, 35

Jackson, J. M., Rathborne, J. M., Shah, R. Y., et al. 2006, ApJS, 163, 145

Jackson, J. M., Finn, S. C., Chambers, E. T., Rathborne, J. M., \& Simon, R. 2010, ApJ, 719, L185

Kim, W.-T., \& Ostriker, E. C. 2002, ApJ, 570, 132

Koyama, H., \& Inutsuka, S.-I. 2000, ApJ, 532, 980

Larson, R. B. 1981, MNRAS, 194, 809

Lee, W.-K., \& Shu, F. H. 2012, ApJ, 756, 45

Leisawitz, D., \& Bash, F. 1982, ApJ, 259, 133

Lockman, F. J. 1989, ApJS, 71, 469

Lynds, B. T. 1970, in The Spiral Structure of our Galaxy, eds. W. Becker, \& G. I.

Kontopoulos, IAU Symp., 38, 26
Mac Low, M.-M., \& Klessen, R. S. 2004, Rev. Mod. Phys., 76, 125

McKee, C. F., \& Ostriker, J. P. 1977, ApJ, 218, 148

Men'shchikov, A., André, P., Didelon, P., et al. 2010, A\&A, 518, L103

Nakanishi, H., \& Sofue, Y. 2006, PASJ, 58, 847

Norman, C. A., \& Ferrara, A. 1996, ApJ, 467, 280

Ntormousi, E., \& Burkert, A. 2011 [arXiv: 1111. 1859]

Pringle, J. E., Allen, R. J., \& Lubow, S. H. 2001, MNRAS, 327, 663

Rathborne, J. M., Johnson, A. M., Jackson, J. M., Shah, R. Y., \& Simon, R. 2009, ApJS, 182, 131

Roman-Duval, J., Jackson, J. M., Heyer, M., et al. 2009, ApJ, 699, 1153

Roman-Duval, J., Jackson, J. M., Heyer, M., Rathborne, J., \& Simon, R. 2010, ApJ, 723, 492

Schinnerer, E., Meidt, S. E., Pety, J., et al. 2013 [arXiv: 1304 . 1801]

Schneider, S., \& Elmegreen, B. G. 1979, ApJS, 41, 87

Shetty, R., \& Ostriker, E. C. 2006, ApJ, 647, 997

Simon, R., Jackson, J. M., Clemens, D. P., Bania, T. M., \& Heyer, M. H. 2001, ApJ, 551, 747

Simpson, R. J., Povich, M. S., Kendrew, S., et al. 2012, MNRAS, 424, 2442

Solomon, P. M., Rivolo, A. R., Barrett, J., \& Yahil, A. 1987, ApJ, 319, 730

Stil, J. M., Taylor, A. R., Dickey, J. M., et al. 2006, AJ, 132, 1158

Tan, J. C. 2000, ApJ, 536, 173

Tasker, E. J. 2011, ApJ, 730, 11

Tasker, E. J., \& Tan, J. C. 2009, ApJ, 700, 358

Thompson, M. A., Urquhart, J. S., Moore, T. J. T., \& Morgan, L. K. 2012, MNRAS, 421, 408

Urquhart, J. S., Hoare, M. G., Purcell, C. R., et al. 2009, A\&A, 501, 539

Van Loo, S., Butler, M. J., \& Tan, J. C. 2013, ApJ, 764, 36

Vázquez-Semadeni, E., Gómez, G. C., Jappsen, A. K., et al. 2007, ApJ, 657, 870

Vázquez-Semadeni, E., Colín, P., Gómez, G. C., Ballesteros-Paredes, J., \& Watson, A. W. 2010, ApJ, 715, 1302

Watson, C., Araya, E., Sewilo, M., et al. 2003, ApJ, 587, 714

Weaver, H. F. 1970, in Interstellar Gas Dynamics, ed. H. J. Habing, IAU Symp., 39,22

Whitworth, A. P., \& Francis, N. 2002, MNRAS, 329, 641

Whitworth, A. P., Bhattal, A. S., Chapman, S. J., Disney, M. J., \& Turner, J. A. 1994, A\&A, 290, 421

Williams, J. P., Blitz, L., \& McKee, C. F. 2000, Protostars and Planets IV, 97

Page 8 is available in the electronic edition of the journal at http://www . aanda. org 


\section{Appendix A: Channel map of the filamentary gas wisp}

In Fig. A.1 we present the channel maps of the ${ }^{13} \mathrm{CO}(1-0)$ emission from the GRS (Jackson et al. 2006) survey. Some contamination from local clouds is indicated.

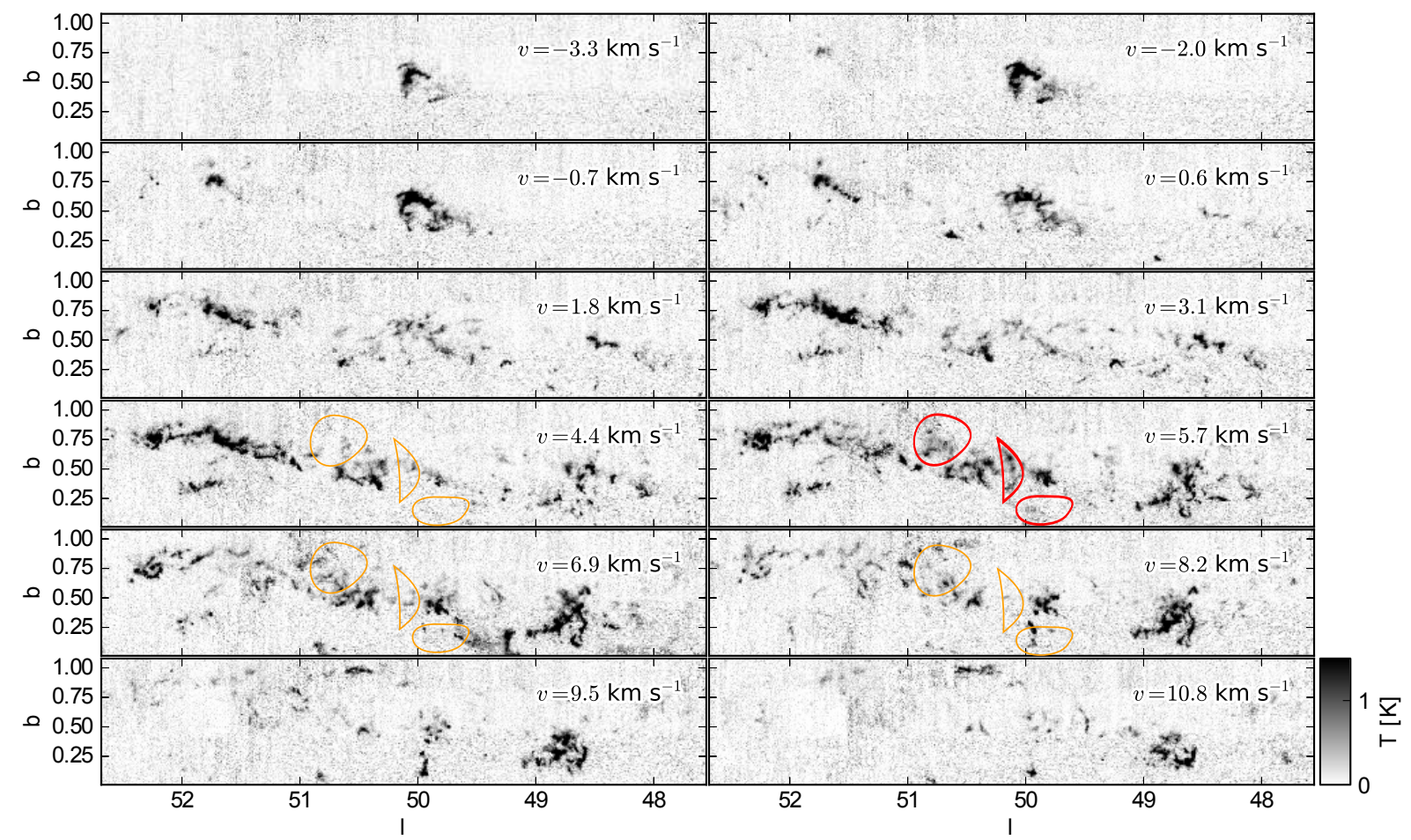

Fig. A.1. Channel maps of the GRS (Jackson et al. 2006) ${ }^{13} \mathrm{CO}(1-0)$ emission of the region. The contaminating gas has a narrow $\left(\sim 0.5 \mathrm{~km} \mathrm{~s}{ }^{-1}\right)$ line width, and is detected only in single channels in the map. Some contamination from local clouds is indicated with red circles as examples, and these red circles are also plotted in yellow in the adjacent velocity channels for comparison.

\section{Appendix B: A comparison with $\mathrm{CO}$ emission from the $\sim 50 \mathrm{~km} \mathrm{~s}^{-1}$ component}

To demonstrate the coherence of our filamentary gas wisp, in Fig. B.1 we present a map of the same region with the velocity integrated within $29.5 \mathrm{~km} \mathrm{~s}^{-1}<v_{\mathrm{lsr}}<73.3 \mathrm{~km} \mathrm{~s}^{-1}$. Seen from the ${ }^{13} \mathrm{CO}(1-0)$ emission, this component is composed of individual patches of molecular clouds and is not as coherent as our filamentary gas wisp. The distance to the region is $\sim 5.3 \mathrm{kpc}$.

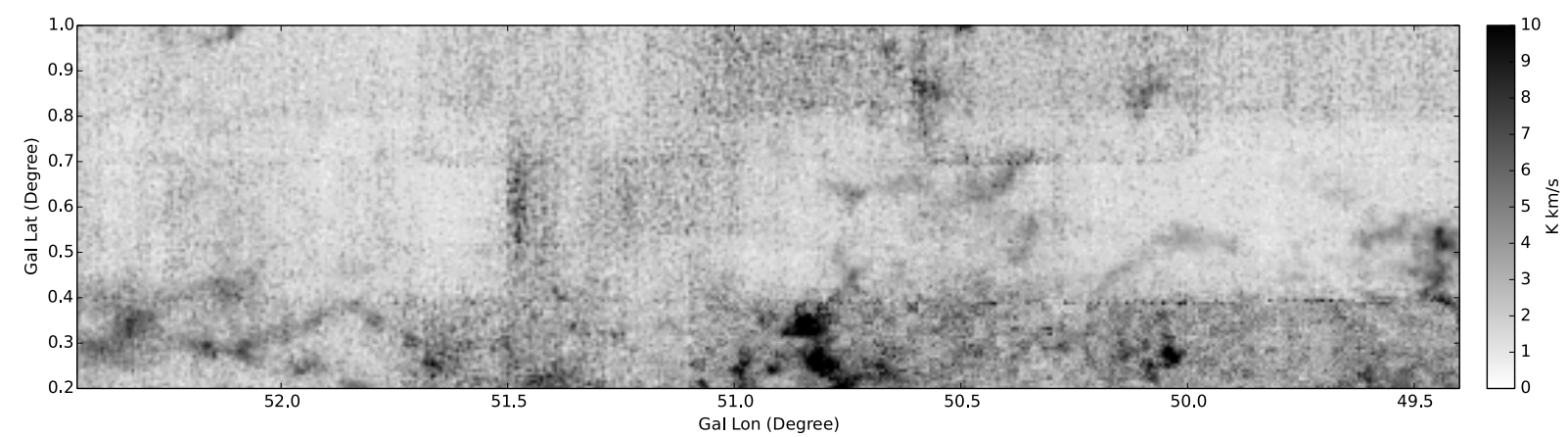

Fig. B.1. Velocity-integrated ${ }^{13} \mathrm{CO}(1-0)$ map of the $\sim 50 \mathrm{~km} \mathrm{~s}^{-1}$ clouds. The emission is from the same region as shown in Fig. 1 and is integrated within $29.5 \mathrm{~km} \mathrm{~s}^{-1}<v_{\mathrm{lsr}}<73.3 \mathrm{~km} \mathrm{~s}^{-1}$. 